\title{
ICRF HEATING ON TFTR WITH THE ORNL ANTENNA*
}

\author{
D. J. Hoffman, W. L. Gardner, and P. M. Ryan \\ Oak Ridge National Laboratory, Oak Ridge, Tennessee 37831 \\ G. J. Greene, J. C. Hosea, J. R. Wilson, and J. E. Stevens \\ Princeton Plasma Physics Laboratory, Princeton, New Jersey 08542
}

\begin{abstract}
Initial ion cyclotron range of frequencies (ICRF) heating experiments on TFTR began in the summer of 1988 . Although we were in the commissioning stage for much of the equipment, some plasma coupling measurements were made in the fail. This paper is focused on the results from the Bay $L$ antenna.
\end{abstract}

\section{INTRODUCTION}

The Bay $L$ antenna comprises a movable array of two loops (designated straps 3 and 4) installed in a 60 - by $90-\mathrm{cm}$ port. It was principally designed to compare, in plasma, a graphite Faraday shield to thin coatings, but it has additional design features ${ }^{1}$ that, in combination with those of the Bay M antenna, were intended to help in the future selection of design options for ICRF antennas. These options include wall slotting vs closed walls, active vs inertial shield cooling, internal vs external matching, vacuum vs $\mathrm{SF}_{6}$ as the high-voltage medium, and copper vs silver as the conducting medium. Although most of these options have not yet been fully tested, some, such as slotting, have been seen to make a significant difference in coupling.2

The plasma response to the Bay $L$ antenna has shown that each strap can effect central heating. Despite the limited operation (a few days of running) and the use of $\mathrm{SF}_{6}$ instead of vacuum on the capacitors, the antennas were powered to $600 \mathrm{~kW}$ on either single strap and $500 \mathrm{~kW}$ on the combined pair. The phased launching is tentatively comparable 2 with that obtained on Bay $M$, but lower power makes it necessary to record incremental plasma performance instead of absolute response.

\section{ANALYSIS OF COUPLING}

The measured power of $600 \mathrm{~kW}$ per strap was achieved for an average power density of $=400 \mathrm{~kW} / \mathrm{cm}^{2}$ flowing through the antenna to the $2 \times 1013 \mathrm{~cm}^{-3}$ plasma located $4 \mathrm{~cm}$ from the face of the antenna. This was seen to be the limit of the antenna at the time, so the circuit needed to be fully analyzed to ascertain whether low-voltage breakdown in the structure or low coupling was responsible. Previous circuit models ${ }^{3}$ using lumped elements implied that the voltage was low $(\approx 30 \mathrm{kV})$. During the vacuum opening, a fully distributed, mutually coupled, lossy coax model was devised for the Bay L antenna (Fig. 1). This model has the expected spatial hyperbolic functions, but has two additional terms that are the result of the mutual inductance between the two straps. The mutual coupling between the two straps is

\footnotetext{
*Research sponsored by the Office of Fusion Energy, U.S. Department of Energy, under contract DE-AC05-84OR21400 with Marin Marietta Energy Systems, Inc.
} 


\section{DISCLAIMER}

This report was prepared as an account of work sponsored by an agency of the United States Government. Neither the United States Government nor any agency thereof. nor any of their employees, makes any warranty, express or implied, or assumes any legal liability or responsibility for the accuracy, completeness, or usefulness of any information, apparatus, product, or process disclosed, or represents that its use would not infringe privately owned rights. Reference herein to any specific commercial product, process, or service by trade name, trademark, manufacturer, or othervise does not necessarily constitute or imply its endorsement, recommendation, or favoring by the United States Government or any agency thereof. The views and opinions of authors expressed herein do not necessarily state or reflect those of the United States Government or any agency thereof. 
included only on the poloidal radiating element. The circuit is reduced to finding appropriate coefficients on the coupled legs from the following equations, assuming that the load, capacitance, and inductance per unit length of each strap are given by $R^{\prime}, C^{\prime}$, and $L^{\prime}$, respectively:

$V_{3}=a_{1} \cosh \left(\Gamma_{+} x\right)+a_{2} \sinh \left(\Gamma_{+} x\right)+a_{3} \cosh \left(\Gamma_{-} x\right)+a_{4} \sinh \left(\Gamma_{-} x\right)$

$V_{4}=a_{1} \cosh \left(\Gamma_{+} x\right)+a_{2} \sinh \left(\Gamma_{+} x\right)-a_{3} \cosh \left(\Gamma_{-} x\right)-a_{4} \sinh \left(\Gamma_{-} x\right)$

$I_{3}=\left(a_{1} / Z_{+}\right) \sinh \left(\Gamma_{+} x\right)+\left(a_{2} / Z_{+}\right) \cosh \left(\Gamma_{+} x\right)+\left(a_{3} / Z_{-}\right) \sinh \left(\Gamma_{-} x\right)+\left(a_{4} / Z_{-}\right) \cosh \left(\Gamma_{-} x\right)$

$\mathrm{I}_{4}=\left(\mathrm{a}_{1} / \mathrm{Z}_{+}\right) \sinh \left(\Gamma_{+} \mathrm{x}\right)+\left(\mathrm{a}_{2} / \mathrm{Z}_{+}\right) \cosh \left(\Gamma_{+} \mathrm{x}\right)-\left(\mathrm{a}_{3} / \mathrm{Z}_{-}\right) \sinh \left(\Gamma_{-} \mathrm{x}\right)-\left(\mathrm{a}_{4} / \mathrm{Z}_{-}\right) \cosh \left(\Gamma_{-} \mathrm{x}\right)$

If the mutual coupling is given by $k$, then

$\Gamma_{ \pm}=\sqrt{ }\left\{\left[R^{\prime}+j \omega L^{\prime} \cdot(1 \pm k)\right] \cdot\left[j \omega C^{\prime}\right]\right\}, Z_{ \pm}=\sqrt{ }\left\{\left[R^{\prime}+j \omega L^{\prime} \cdot(1 \pm k)\right] /\left[j \omega C^{\prime}\right]\right\}$.

At $x=0$ (the input voltage), $V_{3}=a_{1}+a_{3}$, and $V_{4}=a_{1}-a_{3}$. The other two coefficients can be obtained by relating the transformed capacitances at the other end of the two straps.

Circuit parameters were determined through exhaustive correlation of implicit measurements (capacitance values, resonances, and $Q$ to yield circuit parameters), explicit measurements (time domain reflectometry for these parameters), and twodimensional magnetostatic calculations (to confirm the measurements). Table I lists values of every element for strap 3 of the Bay $L$ antenna. Figure 2 shows capacitance vs load per unit length for strap 3's parameters with strap 4 detuned.

\section{IMPLICATIONS OF THE CIRCUIT ANALYSES}

These calculations confirmed that the Bay $\mathrm{L}$ antenna had been conditioned to well beyond $60 \mathrm{kV}$ with vacuum in the capacitors and was limited to $\approx 48 \mathrm{kV}$ with $15 \mathrm{psig}$ of $\mathrm{SF}_{6}$ in the capacitors. At $50 \mathrm{kV}$, the average internal electric field is $43 \mathrm{kV} / \mathrm{cm}$, while that at the ends of the capacitor cans is $1.3 \mathrm{MV} / \mathrm{cm}$. This average field is consistent with the breakdown strength of $\mathrm{SF}_{6}$ at 15 psig.

A more important implication of the distributed model is that the loading is lower than predicted by lumped models. For TFTR discharges of $2 \times 1013 \mathrm{~cm}^{-3}$ plasma located $4 \mathrm{~cm}$ away, the model showed $2.1 \Omega / \mathrm{m}$ for strap 4 and $1.5 \Omega / \mathrm{m}$ for strap 3 , representing relative loads of $\approx 6.1$ and $\approx 4.5$, respectively. This imbalance in loading, unknown during phased operations, complicated analyses. Measurements of the port and antenna location, made during the vacuum opening, show that strap 3 was recessed $=6 \mathrm{~mm}$ from strap 4 due to the port's misalignment. Flux linkage models 3 predict that the recess should reduce the load by $14 \%$, so the imbalance is not completely understood. At this position, the respective power limits were $600 \mathrm{~kW}$ and $390 \mathrm{~kW}$. The power in each strap was limited by the voltage handling limits of the $\mathrm{SF}_{6}$. When reactive loading is included, both antennas operated at $=50 \mathrm{kV}$, the conditioning voltage. Moving the antenna in $8 \mathrm{~mm}$ resulted in a significant gain in power handling ( $550 \mathrm{~kW}$ to $590 \mathrm{~kW}$ ) and a $37 \%$ gain in loading (vs $28 \%$ predicted by the flux linkage model). Thus, the antenna maintained its voltage capabilities despite the increased particle flow.

The model showed two other trends in loading. First, the loading was linearly proportional to the line-average density for ohmic discharges. Increasing the edge 
density (e.g., with neutral beam injection into the discharge) raised the loading. However, this has not yet been quantified, since a systematic study was not made. The second trend is that the loading per strap with two straps is greater than singlestrap loading. Both in-phase and out-of-phase coupling benefited irom this effect. The trend was complicated by the unbalanced loading on the two straps.

\section{PLASMA RESPONSE}

Because the rf power was $\approx 1 / 6$ of the combined if and ohmic power in the discharge, the plasma effects could clearly be seen, but some statistical variations could confuse results. Generally, the heating occurred at the resonance zone. Little impurity introduction was observed. No surface interactions at the face of the antenna, as seen on the Bay $M$ antenna in phase, were detected.

One trend observed on the Bay $L$ antenna, as with the Bay $M$ antenna, is that generally the in-phase operation resulted in $=60 \%$ of the beta increase observed with out-of-phase operation (Fig. 3). On Bay L, when individual straps were powered, the beta increases were comparable to the in-phase rate. Each single strap had the expected heating rate. Because both in-phase and single-strap configurations have some spectral power near the wave numbers of zero, and because the single-strap and out-of-phase configurations are peaked at higher wave numbers than the in-phase configuration, it is suspected that the near-zero spectral wave power is not being usefully transmitted to the core.

\section{FUTURE GOALS AND CONCLUSIONS}

The conclusions of the first runs held some encouragement. The antennas could be operated at good power densities $\left(400 \mathrm{~kW} / \mathrm{cm}^{2}\right)$ and at high voltages $(50 \mathrm{kV})$. The medium in the capacitor was seen to be the voltage limit. Finally, at the $500-\mathrm{kW}$ level, the best core heating was achieved with out-of-phase operation. Single-strap or in-phase operation resulted in a $40 \%$ reduction of efficiency.

Since the detailed analyses led to the conclusion that the loading was low, it is important to try to increase the antenna voltage. Near-term plans include final confirmation of the loading on TFTR. During the vacuum break, the vacuum system for the capacitors has been upgraded so that the capacitors can be pumped by a turbomolecular pump during the magnetic pulse. If the plasma voltages continue to be the same as the conditioning voltages achieved with vacuum in the capacitors, the power should increase by more than $67 \%$. We will also try to facilitate loading by moving the antenna $8 \mathrm{~mm}$ closer to the plasma. Finally, higher-density plasma scenarios in TFTR are planned, including pellet-fueled discharges.

\section{REFERENCES}

1D. J. Hoffman, et al., in Proc. 15th Eur. Conf. Controlled Fusion and Plasma Heating, Dubrovnik, 1988, Vol. 12B, Part II, pp. 770-3 (1988).

2J. Hosea, et al., Bull. Am. Phys. Soc. 33 (9), 2094 (1988).

3D. J. Hoffman, et al., Bull. Am. Phys. Soc. 33 (9), 2094 (1988). 


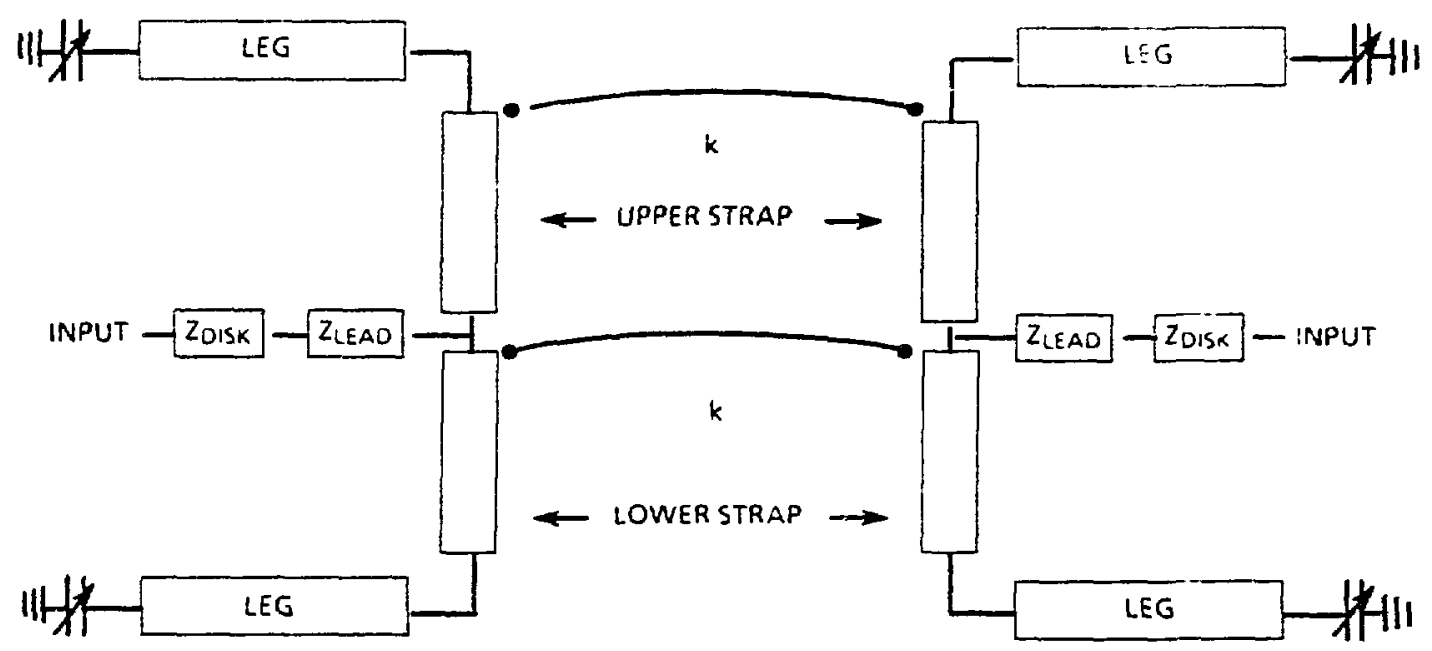

Fig. 1. Circuit diagram of the Bay $L$ antenna.

Table I. Circuit parameters for strap 3

( Tap point $=0.5927$, mutual coupling $=0.02005$ )

\begin{tabular}{lllll}
\hline & Strap & Leg & Disk & Lead \\
\hline Impedance $(\Omega)$ & 42.0 & 75.0 & 17.15 & 60.0 \\
Relative phase velocity $(\%)$ & 66.2 & 99.9 & 34.3 & 100.0 \\
Vacuum losses $(\Omega / \mathrm{m})$ & 0.34128 & 0.010 & 0.010 & 0.010 \\
Length $(\mathrm{m})$ & 0.6775 & 0.4243 & 0.0254 & 0.20 \\
\hline
\end{tabular}

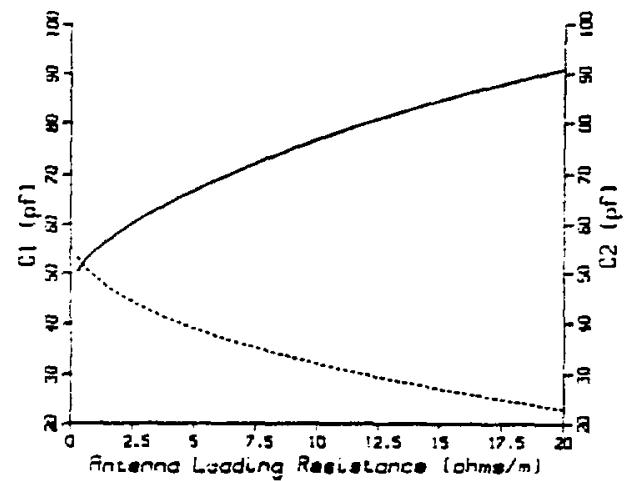

Fig. 2. Capacitance vs load. If the antenna is matched, the capacitance pairs can be used to infer plasma loading.

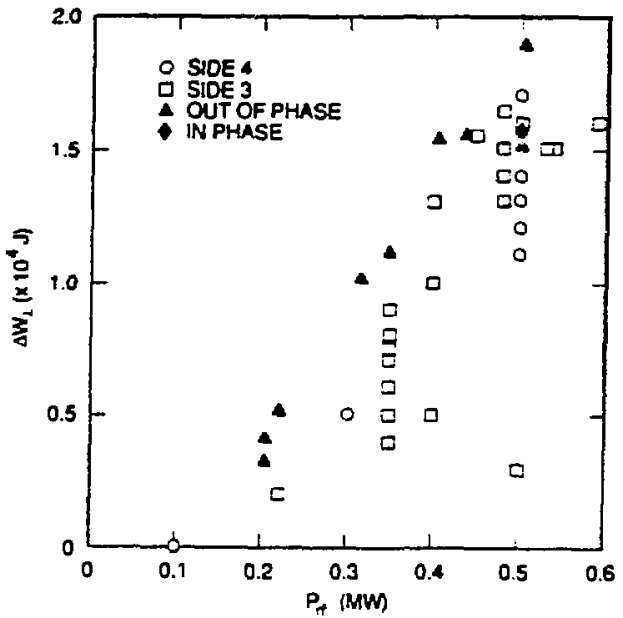

Fig. 3. Change in stored perpendicular energy vs if power for various phasings. 